\title{
An Approach to Abstract Structures of Logistics as a Complex Theory Unifying the Methodology of S-Modelling and the Logic of Science: Initial Steps
}

\author{
Boris Chendov \\ P. O. Box 03, \\ Sofia 1574, Bulgaria \\ E-mail: borischendov@abv.bg, \\ borischendov@yahoo.com
}

\begin{abstract}
In the introduction, following the formulation of the theses (1) on the concept 'philosophy of science', (2) on interdisciplinarity in modern science, and (3) on foundational studies in science, and on the bases of their content, (4) a thesis on the interdisciplinary approach to foundations of science is formulated. In accordance with the latter, together with the canonical approach to foundations of science, which consists in an elaboration of the foundations of mathematics, physics and other fundamental canonical sciences, also an interdisciplinary approach to foundations of science is realised, one that consists in the elaboration of four foundational complex sciences containing the most significant productive factors for the elaboration of various interdisciplinary theories: (1) Methodology of S-modelling, (2) Logic of science, (3) Definitics, a complex theory of definite structures, aggregates, processes and systems in reality and in knowledge, (4) Indefinitics, a complex theory of indefiniteness in its various forms in reality and in knowledge. Taking into account the internal connection between the first two complex sciences enlisted above, it is expedient in the process of investigations of their foundations that they be unified in yet another complicated complex science-logistics.
\end{abstract}

Section 1 outlines the first steps of elaborating the abstract structures of S-modelling methodology. The following three definitions are especially significant in this respect:

Definition 1. We shall say that $\mathbf{X}$ is a system and shall denote it as 'Syst $\mathbf{X}$ ', if and only if $\mathbf{X}$ is a non-empty, in the general case, many-sorted set $\Omega$, supplied with sets of hierarchical (1) predicates $\mathbf{P}$ (properties and substantial relations), (2) functions F, and (3) operations $\mathbf{O}$, closed in $\Omega$. 
Definition 2. We shall say that there is a m-relation of S-modelling of the system $\boldsymbol{X}$ into the system $\mathbf{Y}$ and will write $\mathbf{X m Y}$ if and only if there is at least partial and approximate homomorphism of the system $\mathbf{X}$ into the system $\mathbf{Y}$ in respect to the predicates, functions, and operations.

Definition 3. The system $\mathbf{Y}$ is S-model of the system $\mathbf{X}$ if and only if between systems $\mathbf{X}$ and $\mathbf{Y}$ there is S-modelling relation $\mathbf{X m Y}$.

The System of Successive Spaces (SSS) is introduced as a unified means of S-modelling of mathematical systems with the perspective for further application to S-modelling in the natural sciences.

Section 2 outlines the first steps of elaborating the logic of science. The logic in its well systematised form is treated, first of all, as a system of various logical systems. The basic concept of the logical systems, logical inference, is considerably generalised as concerning the logical acceptance, treated in two basic forms-as value and as relation-as well as on both levels, epistemic and ontological.

Definition 4. Logic of science is a complex science of acceptance-relation (i.e. of logical inference in the most general sense), including philosophicomethodological, proper-logical, historical, and (emphatically) scientificapplied aspects in their mutual connection.

Keywords: foundational studies, interdisciplinarity in science, logical acceptance, logical inference, logical system, logic of science, philosophy of science, S-model, System of Successive Spaces

\section{o. Posing the task of the construction of foundations of logistics}

\subsection{Presuppositions of the present investigation}

The logical start of the present investigation is the task to realise the initial steps to the construction of logistics treated as a complex theory unifying S-modelling methodology and logic of science, revealing its philosophicomethodological foundations. This article is a direct continuation of the topic of my paper presented at the symposium 'Logical and Philosophical Foundations of Science' within the framework of 14 ICLMPS, Nancy, 2011, as well as of my contribution to the round table 'Methodology of Mathematical Modelling and of 
Applications of Logical Systems in Scientific Knowledge' within the framework of the XXIII World Congress of Philosophy, Athens, 2013. Therefore, at the beginning of the present paper I shall formulate an abstract of four theses of the basic results expounded in them, which in their totality play the role of the general methodological presupposition of present investigations.

\section{Thesis 1. On the concept 'philosophy of science'}

1) For the purposes of the forthcoming reasoning we have to make a distinction between the following two aspects of scientific theories and investigations: (1.1) their content and (1.2) their form. Correspondingly, we have to distinguish between the following two types of scientific problems: $\left(1.1^{*}\right)$ ones concerning the content of scientific theories and investigations-the proper problems of science, and $\left(1.2^{*}\right)$ ones concerning their form, determining the approach to the construction of a scientific theory or to the implementation of scientific research - the methodological problems of science.

2) The subject of research in the methodology of science is the class of methodological problems.

3) The class of methodological problems consists chiefly of two groups (or types) of problems: (3.1) problems concerning the language of science which constitute the syntactic and semantic aspects of the methodology of science, and (3.2) problems concerning the methods of raising and solution of (proper) scientific problems which constitute heuristic aspects of the methodology of science.

4) In the course of the historical development of the phenomenon of culture which is usually named 'philosophy', one may reveal two opposite tendencies, which during the $19^{\text {th }}$ and $20^{\text {th }}$ centuries resulted in its splitting into two quite different fields of human knowledge belonging to different spheres of the culture:

4.1) Philosophy of science (Ph. Sc.) —in essence belongs to the sphere of science and at the same time it is a type of knowledge, the main function of which is methodological (i.e. to give methodological prescriptions) in respect to the form of scientific investigations and theories, based on the ontology and epistemology.

4.2) Philosophy of life (in the broadened sense of the term) — consists, in principle, of attitude (cognitive, emotional, behavioural) towards the problems of life which can be expressed by means of different forms as (4.2.1) in theoretical form, belonging to the sphere of the humanities, 
or (4.2.2) by means of various forms of the arts, as well as (4.2.3) by means of various phenomena of the life.

5) In accordance with the formulation under point 4.1, the philosophy of science may be characterised by the following structure:

5.1) Descriptive parts:

(1) Ontology—descriptive models of being on the level of ontological categories

(2) Epistemology—descriptive models of knowledge on the level of epistemic categories.

5.2.) Prescriptive part:

(3) Methodology-prescriptive models of scientific research on the basis of ontological and epistemic models mentioned above under point 5.1 .

6) The structure of philosophy of science outlined under point 5 above revealed that its basis inevitably has to be a system (explicit or at least implicit) of the philosophical categories, treated (6.1) from the epistemic aspect as basic elements of the language of science, presenting themselves limits of semantic analysis of the latter and (6.2) from the ontological aspect as their denotata, presenting themselves in their entire system the fundamental definiteness of reality (in addition, we have in mind the latter as containing essential elements of indefiniteness). From this statement immediately follows the conclusion concerning the essential role of the system of categories for the unification of the grounds of a system of philosophy of science.

7) These kinds of problems — ontological, epistemological and methodological— arise in various fields of science in a concrete form, closely connected with the process of their historical development, and frequently subjected to separate discussions, but in essence they form a theoretical system possessing its (1) special topic of research, (2) system of basic concepts, (3) scope of problems, (4) arsenal of research methods; and this fact in particular (expressed by 1-4) motivates the genesis of philosophy of science as a separate theoretical system in the framework of science.

8) So, we use the terms 'philosophy of science' and 'philosophy of life' as denotations of correspondingly two essentially different explications of the essentially indefinite (in semantic respect) term 'philosophy'.

In order to avoid any misunderstanding I shall explicitly formulate that one may say that science can be a topic of consideration for both philosophy 
of science and philosophy of life, but in essentially different ways as well as with different subject, value and importance, and correspondingly to the difference between science and the humanities. So, the treatment of science in the framework of the philosophy of science has to occur in a strictly scientific way, which on its part requires limitation of the subject of its treatment by means of its given explication, expressing its given, well-defined aspect, while the treatment of science in the framework of philosophy of life, on the contrary, allows a very large scope of its aspects to be subjected to contemplation, containing intuitive, visual, emotional and, possibly, also some rational components. Besides, it has to be stressed that such a contemplation can turn out to be very reasonable and useful, however it cannot be evaluated as scientific.

9) For a philosopher of science, the most important activity is not purely theoretical one directed to the elaboration of a good, very abstract, wellordered and closed rational system of statements concerning ontological, epistemological and methodological problems of science. Indeed, such a pure theoretical activity is also important for a philosopher of science, even if it is very important, but in any case for him it is auxiliary and, hence, of secondary significance. The most important activity for a philosopher of science is the practical (in respect to philosophy of science) activity directed to the research of specific scientific problems belonging to one or other branch of science on the foreground of its development, however from the philosophical point of view, he is ultimately restricted to elaborating the approach to research of the given problems.

At the same time, these two distinct forms of activity of the philosopher of science are closely connected and they alternate with one another in the historical process of development of science: (1) the pure theoretical activity directed at the elaboration of a good, well-ordered system of philosophy of science provides the philosopher of science with a substantial theoretical basis for his investigations of concrete scientific problems; (2) the results of the latter supply the philosopher of science with raw concrete materials for generalization in the process of elaboration of abstract system of philosophy of science.

Thesis 2. On the interdisciplinary tendency in modern science

1) With the publication of Cybernetics by Norbert Wiener in 1948, a new (third) period in the history of science began, the distinguishing feature of which is the tendency of the domination of interdisciplinary sciences, theories and 
investigations equally with canonical sciences, theories and investigations (such as mathematical, physical, chemical, biological, and the like).

2) There are mainly two types of interdisciplinary sciences:

2.1) Boundary sciences and theories, which subject involves objects and phenomena on the boundary between two qualitatively different types of objects and phenomena, correspondingly treated in two adjacent canonical sciences (for example, physical chemistry as a science on the boundary between theoretical physics and chemistry, or biochemistry as a science on the boundary between chemistry and biology).

2.2) Complex sciences and theories, which subject of study are complex objects (in the large sense involving in its scope also complex phenomena), the different parts and aspects of which are the subject of study in the framework of different canonical sciences. They are divided into two classes:

2.2.1) Complex sciences and theories based on abstract objects (which can be called also complex theories of first order) — the complex objects which serve as subject of research in this case are abstract structures (resp. systems) together with their more various concrete interpretations corresponding to different branches of canonical sciences.

2.2.2) Complex sciences and theories of concrete objects (which can be called also complex sciences and theories of second order), which have to deal with the all-embracing investigation of a given concrete object or type of objects distinguished as very complicated (as, for example, the earth).

3) At the contemporary stage of the history of science, the most fundamental change in the structure of classification of science is made by the complex sciences, which presuppose an essentially new type of classification of scientific problems being synthesis of various canonical scientific problems. Boundary sciences and theories, on the contrary, take place in the canonical classification of sciences, besides they are expanded with overlapping boundary areas between various fundamental sciences for corresponding boundary sciences revealing an essential connection between them-for example, the overlapping boundary area between physics and chemistry for physical chemistry, the overlapping boundary area between chemistry and biology for biochemistry, etc.

4) The connection between more abstract and general elements (concepts, problems, propositions, methods) of the philosophy of science, on the 
one hand, and relatively more concrete and specified elements (concepts, problems, propositions, methods) of the corresponding special science, on the other hand, obtains an internal and immediate character in interdisciplinary investigations, especially in the complex sciences and theories.

\section{Thesis 3. On foundational studies in science}

1) During the $19^{\text {th }}$ and the first half of the $20^{\text {th }}$ century, a new type of scientific investigations began to develop side by side with experimental, theoretical (in the narrow sense) and philosophical ones-foundational studies (in German, Grundlagenforschung). One can distinguish in this process the following three basic stages:

1.1) During the $19^{\text {th }}$ century and the first four decades of the $20^{\text {th }}$ century in the course of enrichment of mathematics with an abundance of new concrete results also foundational studies were gradually being developed in it-first in the field of mathematical analyses (Caushy in the 1830s and Weierstrass in the 1860s and 1870s), subsequently in the field of the theory of real numbers as a basis of mathematical analysis (Dedekind), in the field of the theory of natural numbers (Cantor in 1872-1898, Frege in 1879-1903, and Peano in 1889), in the field of geometry (Hilbert in 1899), in the field of logic and arithmetic (Hilbert in 1904), in the field of set theory (Zermelo in 1908, Fraenkel in 1922), in the field of probability theory (Richard von Mises in 1919-1928 and A. N. Kolmogoroff in 1933).

1.2) In the 1920s and 1930s, David Hilbert made an essential step towards generalisation of the foundational studies in mathematics by making a transition from studies of foundations of one or another branch of mathematics, such as geometry, arithmetic and so on, to foundations of all mathematics, otherwise speaking to foundations of mathematics in its integrity. This step was manifested by him by means of both the content and terminology of a series of papers published manly during the 1920s_-Neubegründung der Mathematik' (Hilbert, 1922), 'Die logischen Grundlagen der Mathematik' (Hilbert, 1923), 'Die Grundlagen der Mathematik (Hilbert, 1928), 'Probleme der Grundlegung der Mathematik' (Hilbert, 1929), and later by the fundamental monograph in two volumes, Grundlagen der Mathematik, published together with Paul Bernays (Hilbert \& Bernays, 1934 \& 1939).

The term 'foundation of mathematics' was applied also to other directions of fundamental treatments of mathematics: cantorism by 
Cantor; logicism by Frege, Whitehead and Russell, intuitionism by Brouwer and Herman Weyl (in addition, Hilbert's way of elaboration of foundations of mathematics obtained the name 'formalism'). At the same time it is worth mentioning that these key-terms 'cantorism', 'logicism', 'intuitionism', and 'formalism' are used also as denotations of the principal positions of the corresponding authors on the problems of philosophy of mathematics. This fact of linguistic character is a demonstration of the essential circumstance that foundations of mathematics turned out to be actually formed as an interdisciplinary field of science, more precisely, a boundary science between philosophy of science and mathematics, containing two basic components: (1) 'philosophy of mathematics', and (2) logically fundamental elements of mathematics. This state of affairs is distinctively expressed in the excellent monograph The Foundations of Mathematics by Evert Beth, who stressed in its preface to the first edition dated February 1959: "The present work is designed to combine [...] treatment of both philosophical and logico-mathematical problems" (Beth, 1968, p. x).

The development of foundations of mathematics during the $20^{\text {th }}$ century was crowned with great success as it enriched considerably with new results both mathematics and philosophy of mathematics (and, at the same time, the philosophy of science).

1.3) The convincing success of the work in the field of foundations of mathematics naturally led to the question about the elaboration of foundations of physics, biology and so on, following the example of foundations of mathematics, and further to take the next step of generalisation of foundational studies, thus realising a transition from foundations of the various fundamental sciences such as mathematics, physics, biology and so on to foundations of all science, in other words, to foundations of science in its integrity, or merely to foundations of science.

The task of elaborating the foundations of science (as a whole), including also the task of separate elaboration of various fundamental sciences, was posed during the 1930s by Otto Neurath, one of the most active members of the Vienna circle, in the form of his advanced idea to compose a modern Encyclopedia of Unified Science. In accordance with the initial design of Neurath, the very complicated and difficult work on realisation of this task was preceded by a two-volume book entitled Foundations of the Unity of Science. I suppose this work (the first edition was published in 1938, and the following editions consecutively supplemented in order to realise more 
completely the initial plan of the book in 1939, 1946, 1951, 1952, 1955, and 1969) can be accepted historically as the first essential attempt to present a system of foundations of science, involving also attempt of presentation of foundations of some fundamental fields of the science-I have in mind the monographs in its framework: Foundations of Logic and Mathematics by Rudolph Carnap, Foundations of the Theory of Signs (in a contemporary terminology, foundations of semiotics) by Charles Morris, Foundations of Physics by Philipp Frank, and Foundations of Biology by Felix Mainx (Neurath, Carnap \& Morris, vol. I, 1938, 1969). Unfortunately, this came to a stop with the death of the initiator of this activity, Otto Neurath, on 22 December 1945 . Nevertheless, the task posed by Neurath in essence does not lose its importance for further development of science.

2) Taking into account the historical facts formulated above under point 1 , functioning as empirical data here for philosophical generalizations, we may formulate and accept the following two statements:

2.1) The basic aim of foundational studies consists (1) in the substantiation of scientific knowledge, involving (1.1) to be guaranteed in such a degree as possible inadmissibility of any internal or external (in respect to the given theoretical system) contradictions, respectively paradoxes, and (1.2) to provide it with explicit strong argumentation of the accepted propositions in the corresponding form; (2) supply of the scientific knowledge with convenient and effective methods of research, and (3) unification of the various elements of the given theoretical system-every one of these items was realised actually in the course of historical process of formation of foundations of science considered under point 1 .

2.2) Foundational studies and, at the same time, corresponding systems of foundations of science (on three levels: (1) of the science in its integrity, (2) of the fundamental sciences, and (3) of the branches of last ones) consist of two basic components: $\left(1^{*}\right)$ a fragment of the philosophy of science, and $\left(2^{*}\right)$ a logically fundamental fragment of the corresponding science.

3) There is an essential internal connection between these two components of the foundations of sciences mentioned above under point 2.2: (1) the first component consisting in a fragment of philosophy of science by virtue of its methodological function lays the foundation and gives direction to the elaboration process of the principal propositions of the corresponding part of the science, constituting the content of the second component by means 
producing chiefly (1.1) the exact language of science and (1.2) substantiated ways of posing and solving scientific problems; so, to put it briefly, the first component obtains application and together with this also realisation in the process of elaboration of the second component; (2) the second component (2.1), firstly, in accordance with the general rule that the following step in the process sheds light on the semantic meaning and the significance of the previous step, facilitates additional elucidation of the semantic meaning and the significance of the various elements of the philosophy of science as the first component of the given foundations, (2.2) secondly, containing principal elements of the given field of science determining in general its many-sided development, depending on its success or failure in this respect serves as a criterion for evaluation of these parts of the philosophy of science which constitute the content of the first component and, by doing that, exerts influence back to its philosophico-methodological basis in a positive or negative way.

4) Taking into account the two-component structure of the foundations of science (for the three levels of science) mentioned under point 2.2), as well as the essential internal connections between these two components, expressed under point 3), we can draw the conclusion that the various foundations of science (in their various forms) are of interdisciplinary character and, more precisely, they are boundary sciences, positioned between a fragment of philosophy of science and a corresponding fragment of the special science.

5) From the thesis concerning the interdisciplinary character of the various types of the foundations of science specified more precisely as boundary sciences, formulated above under point 4 , at least the following two consequences follow:

5.1) It is reasonable to expand the meaning of the concepts 'approach' and 'methodology', which initially were used here only in respect to philosophy of science (see the beginning of the article, Thesis 1), attributing to them the foundations of science in their entirety. Accordingly, we have to make a distinction between philosophical approaches and methodology, on the one hand, and special scientific approaches and methodologies, on the other, which are represented by means of logically significant elements of a given theoretical system (as for example by means of axiomatic systems).

5.2) As a consequence of the narrow and internal connections between both components of the foundations of science mentioned under point 3 , the philosophy of science acquires the most developed form and at 
the same time the most effective methodological significance for the respective part of scientific knowledge when it serves the role of the first component of corresponding foundations of science.

Thesis 4. On interdisciplinary approach to foundations of science

1) Taking into account what was mentioned in Thesis 2 on the interdisciplinary tendency in modern science and in Thesis 3 on foundational studies in science, we first have to raise Neurath's idea for the elaboration of foundations of the unity of science as a very pressing matter in the era of interdisciplinary investigations; secondly, however, we essentially have to revise his conception concerning the unity of science: together with elaboration of the unity of canonical sciences, which Neurath actually had in mind (using our terminology introduced above) — the unity of mathematics, physics, biology, etc., we have to elaborate also the unity of interdisciplinary sciences and at the same time together with the canonical approach to foundations of science we also have to realise an interdisciplinary approach to foundations of science.

2) Taking into account some considerations concerning the structure of scientific theories and investigations, the author of the present work accepts that the theoretical presentation of the foundational kernel of interdisciplinary sciences consists of the following four complex sciences of first order (in the sense formulated in Thesis 2, point 2.2.1) containing the fundamental theoretical productive factors for elaboration of the interdisciplinary sciences and theories:

(1) Methodology of S-modelling,

(2) Logic of science,

(3) Definitics (a complex theory of definite structures, aggregates, processes and systems in reality and in knowledge),

(4) Indefinitics (a complex theory of indefiniteness in its various forms in reality and in knowledge).

3) Taking into account the internal connection between the first two complex sciences enlisted above, it is expedient in the process of investigations of their foundations that they be unified in one more complicated complex science (i.e. a complex science of the second order)—-logistics; starting from similar considerations it is expedient in the process of investigations of foundations of the last two complex sciences enlisted above to be unified in one more complex science (i.e. also in a complex science of the second order)—predicamentics. 
4) Each of the foundational complex sciences (1)-(4), mentioned above under point 2, contain elements belonging to the rest of the three sciences from the given list; consequently, it contains elements of modelling, logical and philosophical foundations of science. At the same time one may say that (1) elements of modelling foundations of science predominate in the Methodology of S-modelling and, accordingly, it can be treated as containing the kernel of the modelling foundations of science; (2) elements of logical foundations of science predominate in the Logic of Science and, accordingly, it can be treated as containing the kernel of the logical foundations of science; (3) elements of philosophical foundations of science predominate in the Predicamentics and, accordingly, it can be treated as containing the kernel of the philosophical foundations of science.

\subsection{The order of the task of the present investigation}

1) Taking into account Theses $1-4$ formulated above and accepting them as the philosophico-methodological presuppositions of the present investigation, we have to formulate here the initial steps of construction of the logistics treated as a complex science of the second degree (of complexity), unifying the following two complex theories of the first degree (of complexity) - (1) the methodology of S-modelling and (2) the logic of science, and playing the role of foundational basis for construction of various interdisciplinary sciences and theories.

2) Thesis 5. In logistics, one can distinguish between the following two basic groups of problems:

2.1) problems concerning a systematic description of abstract structures of S-modelling theory and of the logic of science in their mutual connection and integrity-to some degree they correspond to foundations of mathematics (but do not coincide with the latter);

2.2) problems concerning the systemic application of the abstract structures of the $\mathrm{S}$-modelling theory and of the logic of science to the construction of various theories in the field of mathematics and natural sciencesto some degree they correspond to theoretical elaboration of the corresponding problems of mathematics and natural sciences.

Note to Thesis 5. It is obvious that first we have to treat the first group of problems mentioned above and next on the basis of the obtained results it is possible to treat the second group of problems. At the same time we have to mention that in the course of the treatment 
of problems of the second group some new, additional problems and ideas concerning the abstract structures of the $S$-modelling theory and of the logic of science may appear.

3) Taking into account Thesis 5 , the task of the present investigation will be limited to the treatment of the first group of problems - those concerning the abstract structures of the S-modelling theory and of the logic of science, preparing in such a way the basis for the treatment of the second group of problems to be mentioned in a further research publication. Besides, in light of the very limited scope of the present paper, the treatment of this topic will be restricted here merely to a description of the initial steps of construction of S-modelling theory and of the logic of science, which reveals a philosophico-methodological approach to the present topic.

4) According to a presently wide-spread point of view, the basis of contemporary mathematics is the set theory. From the aspect of this current approach to the problems of abstract structures of mathematics we have to assume that the latter are based on the basic abstract structures of S-modelling theory and of the logic of science, i.e. the basic abstract structures of logistics. This statement does not exclude the role of the set theory in the foundations of mathematics, however it changes this role as a part of the complicated and more profound role of S-modelling theory in the foundations of mathematics.

\section{Abstract structures of the S-modelling theory-initial steps of their elaboration}

\section{Preliminary notes}

\section{1) The motives for introducing the concepts of S-model and S-modelling}

The words 'model' and 'modelling' are used in the natural languages and their meaning varies depending on their application in various sciences. Since the second half of the $19^{\text {th }}$ century the terms 'model' and 'modelling', and later 'mathematical model' and 'mathematical modelling' were increasingly adopted as elements of the language of various branches of science; recently, however, their use is connected with the manifestation of an essential form of an interdisciplinary approach to the investigation of scientific problems. At the same time, however, these terms are used with various degrees of precision and 
in essence they acquire different meanings and are treated methodologically differently in different fields of scientific research. Moreover, in some cases one may state even opposite semantic and methodological treatment. For example, the modelling economic systems and processes by means of algebraic equations is usually apprehended as a way of formalization of theoretical and practical economics. A similar situation takes place also in other empirical fields of science and humanities. In the logic, however, modelling is apprehended and treated as a way of transition of the thought in the opposite direction: from pure formal level of research to conceptual (in German, inhaltlich) level of logical research. Such a discrepancy is a serious hindrance to interdisciplinary communications and investigations. So, it is urgent to pose the task for elaboration of a unified general theory of models and modelling, based on the general definition of the basic concepts 'model' and 'modelling' and destined for application in various fields of science and particularly in the field of interdisciplinary investigations.

Indeed, having in mind the general methodological requirement to the language of science to be (1) exact, and at the same time (2) heuristically potent for the elaboration of scientific theories, as well as complying with (3) the specific methodological requirement of interdisciplinary investigations for unification of the common parts of languages of various canonical sciences (as a necessary condition to make the fruitful communication between representatives of different canonical sciences possible), we have to work out appropriate unified explications of the so far intuitively used notions of model and modelling in the various fields of science. Besides, such a unified explication of the general concepts of models and modelling is a necessary condition for elaboration of a General Theory of Models and Modelling which has to serve as general, unified basis for the treatment of problems about modelling, in various fields of science-canonical as well as in ones of interdisciplinary type.

\section{2) The way to explicate the intuitive notions of models and modelling}

Taking into consideration the principal distinction between intuitive concepts and their explications in the language of science it is clear that a thorough empirical analysis of the way the concepts 'model' and 'modelling' are used in the course of scientific communications and investigations can turn out to be very fruitful for heuristic purposes of inventing a class of possible appropriate explications of them, but presently there seems to be no possibility of strong derivation (i.e. of deduction) of their actual explication from the results of such an empirical analysis. Consequently, when using the results of such an empirical analysis merely on the heuristic level we ultimately have to postulate explications of the intuitive notions of models and modelling and further, on the basis of 
these hypothetically accepted explications, make an attempt to construct a general theory of models and modelling. In addition, depending on the success or failure of this attempt, it will ultimately lead to a full acceptance, partial acceptance or rejection of the corresponding explications.

For the sake of brevity I shall omit here the exposition of such an empirical analysis of heuristic significance and shall directly postulate explications of the intuitive notions of models and modelling.

\subsection{Preliminary definition of the concept of S-model}

Roughly speaking, the concept 'S-model' concerns a relation (named m-relation, or modelling relation) between two systems, called 'system-object', $\mathbf{S}_{O b j}$, and 'system-model', $\boldsymbol{S}_{\text {Mdl }}$. So, the term 'S-model' (or System-model) will be denoted by the triplet $<\mathbf{S}_{O b j}, \mathbf{S}_{M d l}, \mathbf{m}>$.

\subsection{On the concept of system}

1) Df. Syst $(\mathbf{X})$. We shall say that $\mathbf{X}$ is a system and shall denote it as 'Syst $(\mathbf{X})$ ', if and only if $\mathbf{X}$ is a non-empty, in the general case many-sorted set $\Omega$, supplied with sets of hierarchical (i.e. of varying degrees) (1) predicates $\mathbf{P}$ (properties and substantial relations), (2) functions $\mathbf{F}$, and (3) operations $\mathbf{O}$, closed in $\Omega$ :

$$
\operatorname{Syst}(\mathrm{X})=<\Omega,\left\{\mathrm{P}_{j}\right\},\left\{\mathrm{F}_{k}\right\},\left\{\mathrm{O}_{l}\right\}>,
$$

where $\Omega \neq \varnothing, \mathbf{j}, \mathbf{k}, \mathbf{l} \in \mathbf{N}_{\mathbf{1}}, \mathbf{N}_{\mathbf{1}}$ is a set of indexes denoting the natural order of integers beginning with $1: 1,2,3, \ldots$.

Note 1 to Df. Syst $(\mathbf{X})$. The formulation above of the preliminary definition of the concept of system $\mathbf{X}$ presupposes a distinction between two kinds of relations, which will be conditionally named here as follows: (1) formal relations - these are binary relations which bring in correspondence some element of $\Omega$, say $\mathbf{a}, \mathbf{a} \in \Omega$, with some element $\mathbf{b}$, also $\mathbf{b} \in \Omega,(\mathbf{a}=\mathbf{b}$ is allowed), so it is irrelevant in respect to the nature of its elements; and (2) substantial relations - these are relations relevant to the nature of its elements like relations of determinations of various kind, relations between parents and children, and so on.

The cases in the language of mathematics when operations are treated as functions and functions as relations are connected with $\left(1^{*}\right)$ treatment of relations as formal ones (in the sense mentioned above) and $\left(2^{*}\right)$ using extensional approach to the treatment of concepts connected 
with identification of extensionally equal concepts. Correspondingly, $\left(1^{* *}\right)$ distinction between formal and substantial relations realised above and $\left(2^{* *}\right)$ using intentional approach to concepts connected with distinction between intentionally different concepts even in the cases in which they are extensionally equivalent allows us to speak about predicates, functions, and operations as about different entities.

Note 2 to Df. Syst $(\mathrm{X})$. Roughly speaking, under "hierarchical (i.e. of varying degrees) (1) predicates $\mathbf{P}$ (properties and substantial relations), (2) functions F, and (3) operations $\mathbf{O}$ " in Preliminary Df. Syst X, we shall understand such predicates, functions, and operations which are distinguished between different degrees $1,2,3$, etc. in such a way that for those of the first degree the field of their arguments and values are only elements of the basis of a given system $\Omega(\Omega=\mathbf{B} \boldsymbol{s}(\mathbf{X}))$; for those of the second degree, the field of their arguments and values is obtained as a result of enlargement of the field of arguments and values of the predicates, functions, and operations of the first degree, by means of including to it subsets of $\Omega$, as well as predicates, functions, and operations of the first degree; further for those of the third degree the field of their arguments and values is obtained as a result of enlargement of the field of arguments and values of the predicates, functions, and operations of the second degree by means of including to it also sets of subsets of $\Omega$, as well as predicates, functions, and operations of the second degree, and so on. If we give to this reasoning a strong form of inductive definition we shall obtain a complete definition of the concept "hierarchical (i.e. of varying degrees) (1) predicates $\mathbf{P}$ (properties and substantial relations), (2) functions $\mathbf{F}$, and (3) operations $\mathbf{O}$ ".

2) Df. $\mathbf{B s}(\mathbf{X}), \operatorname{Str}(\mathbf{X})$. The set $\Omega$ in the equation $\{1\}$ will be called "the basis of the system $\mathbf{X}$ " and will be denoted as 'Bs $(\mathbf{X})$ '; the ordered set in it

$$
<\left\{\mathrm{P}_{j}\right\},\left\{\mathrm{F}_{k}\right\},\left\{\mathrm{O}_{l}\right\}>
$$

will be called "structure of the system $\mathbf{X}$ " and will be denoted as ' $\operatorname{Str}(\mathbf{X})$ '.

3) Df. vSyst $(\mathbf{X})$. We shall say that $\mathbf{X}$ is a very system (i.e. "that $\mathbf{X}$ is a system in the narrow sense") and shall denote it as ' $\mathbf{v S y s t}(\mathbf{X})$ ' if and only if

$$
(\Omega \neq \varnothing) \&\left(\left(\left\{\mathrm{P}_{j}\right\} \neq \varnothing\right) \mathrm{V}\left(\left\{\mathrm{F}_{k}\right\} \neq \varnothing\right) \mathrm{V}\left(\left\{\mathrm{O}_{l}\right\} \neq \varnothing\right)\right) .
$$

4) Df. sSyst $\mathbf{X}$. We shall say that $\mathbf{X}$ is a semi-system (i.e. "that $\mathbf{X}$ is a system in the non-proper sense") and shall denote it as 'sSyst(X)' if and only if 


$$
(\Omega \neq \varnothing) \&\left(\left\{\mathbf{P}_{j}\right\}=\varnothing\right) \&\left(\left\{\mathbf{F}_{k}\right\}=\varnothing\right) \&\left(\left\{\mathbf{O}_{l}\right\}=\varnothing\right) .
$$

Note to Df. sSyst $(\mathrm{X})$. It is obvious that the concepts 'semi-system' and 'nonempty set' are extensionally equivalent, but intentionally different:

(X is sSyst) if and only if $((\mathrm{X}=\Omega) \&(\Omega \neq \varnothing))$,

i.e. $\mathbf{X}$ is system in non-proper sense if and only if $\mathbf{X}$ is non-empty set.

\subsection{On the concepts of S-model and of S-modelling}

1) Df. S-Mdrel $\mathbf{m}$. We shall say that there is m-relation ( $S$-modelling relation) of the system $\boldsymbol{X}$ into the system $\boldsymbol{Y}$ and will write ' $\mathrm{XmY}$ ' if and only if there is at least partial and approximate homomorphism of the system $\mathbf{X}$ into the system $\mathbf{Y}$ in respect to the predicates, functions, and operations.

Note to Df. S-Mdrel $\mathbf{m}$. In such a case the system $\mathbf{X}$ plays the role of an object-system and the system $\mathbf{Y}$ the role of the modelling system, i.e. $\boldsymbol{S}_{O b j}=\mathbf{X}$, and $\mathbf{S}_{M d l}=\mathbf{Y}$.

2) Df. S-Mdl. The system $\mathbf{Y}$ is an $S$-model of the system $\mathbf{X}$ if and only if between systems $\mathbf{X}$ and $\mathbf{Y}$ there is S-modelling relation $\mathbf{X m Y}$ :

$$
\mathrm{Y}=\mathrm{S}-\mathrm{Md}(\mathrm{X}) \leftrightarrow \mathrm{XmY}
$$

3) Df. pure S-Mdl. The subsystem $Y^{\prime}$ of the system $Y, Y^{\prime} \subseteq Y$, will be called pure S-model of the system $\mathbf{X}$ (resp. proper modelling system in respect to the system $\mathbf{X}$ ) if and only if it contains only those elements, predicates, functions, operations of $\mathbf{Y}$, which are put in correspondence with elements, predicates, functions, operations of $\mathbf{X}$, respectively, in the homomorphism XmY.

4) Df. ismS-Mdrel. We shall say that there is an isomorphic $S$-modelling relation (abbreviated as: ism-relation) of the system $\boldsymbol{X}$ into the system $\boldsymbol{Y}$ and will write 'XismY' if and only if there is at least partial and approximate isomorphism of the system $\mathbf{X}$ into the system $\mathbf{Y}$ in respect to the predicates, functions, and operations.

In such a case the system $\mathbf{X}$ can play the role of an object-system and the system $\mathbf{Y}$ the role of the modelling system, and vice versa.

5) Df. ismS-Mdl. The system $\mathbf{Y}$ is isomorphic S-model of the system $\mathbf{X}$ if and only if between systems $\mathbf{X}$ and $\mathbf{Y}$ there is isomorphic S-modelling relation:

$$
(\mathrm{Y}=\mathrm{ismS-Mdl}(\mathrm{X})) \leftrightarrow \mathrm{X} \text { ism } \mathrm{Y}
$$


in such a case we shall say also "the system $\mathbf{Y}$ is a complete model of the system X".

6) Df. S-Mdg. We shall call the process of S-modelling the totality of the processes of (1) construction, (2) investigation and (3) application of S-models.

Note to Sections 1.2 and 1.3. The systems and S-models as defined in section 1.2, point 1) and in section 1.3, point 2), respectively, can be subjected to algebraic treatment and in such a way we obtain the capacity to construct algebra of systems and of S-models.

\subsection{Theses on S-models and S-modelling}

1) Th. of variety of S-Mdl and S-Mdg. (1) Depending on the variety of concrete forms of the (1.1) object-systems, (1.2) modelling systems and (1.3) m-modelling relations, (2) there is a great variety of corresponding forms of S-models; in addition, (3) depending also on the variety of concrete forms of the (3.1) formation, (3.2) investigation and (3.3) application of $\mathrm{S}$-models, (4) there is a great variety of corresponding forms of the processes of S-modelling.

Note to Th. of variety of forms of S-models and of S-modelling. The thesis of variety of S-models and of S-modelling is formulated above in order to resist the wide-spread tendency of specialists of various special sciences speaking about methodological problems of scientific modelling to take into consideration only a very limited class of models as well as of forms of procedure of scientific modeling which are usually used and well known in their proper field of investigation, while at the same time ignoring the sheer vastness of the variety of models and of forms of procedure of scientific modeling which are used in other disciplines. Such a limited way of approaching to the general methodological problems of scientific modeling results in a formulation of one-sided misconceptions of models and modelling in scientific knowledge.

2) Th. ismS-Mdl $(\mathrm{Y}=\operatorname{ismS-Mdl}(\mathrm{X})) \rightarrow((\mathrm{Y}=\mathrm{S}-\mathrm{Mdl}(\mathrm{X})) \&(\mathrm{X}=\mathrm{S}-\mathrm{Mdl}(\mathrm{Y})))$

3) Th. Ont of S-Mdrel and S-Mdg. (1) The relations of S-modelling between some natural systems, as well as (2) the processes of S-modelling in the form of natural process of formation of model relations between natural systems 
(3) take place among various systems in reality and (4) play an essential role in the definiteness of the reality.

4) Th. ScTh as S-Mdl. Every scientific theory (ScTh) developed in a sufficient degree is in essence (nevertheless in explicit or at least in implicit form) (1) a kind of S-model of its subject of research (2) involving (2.1) definitions of concepts, (2.2) formulations of tasks (resp. of problems), (2.3) theses (in the form of theorems, scientific laws, etc.) as solutions of corresponding problems, (2.4) prescriptions (especially in the forms of rules), (2.5) arguments (in particular, strong logical proofs of theorems in mathematics, theoretical and empirical demonstrations of laws in physics, etc.), and (2.6) methods for posing and solving scientific problems, respectively, tasks, so that (3) the cognitive elements mentioned under point 2 are connected between themselves in such a way that they are unified in systematic theoretical wholes (4) by means of relation of determination between them-(4.1) by means of logical consequences between them and (4.2) on the basis of causal relationship in the corresponding subject of research.

5) Th. DvlSc as S-Mdg. The processes of development of science (DvlSc) is a process of development of constructions, investigations and applications of S-models (in other words, a process of development of S-modelling processes, see Section 1.3, point 6).

6) Th. Epist as S-MdgTh. The epistemology (Epist) in the framework of the philosophy of science treated as philosophical theory of scientific knowledge can be regarded as a partial case of the more general and on a larger scale all-embracing theory of S-models and S-modeling (S-MdgTh).

7) Th. Tr in S-Mdl. (1) The truth (Tr) and falsehood of a given statement in the given scientific theory treated in itself is of analytical character, i.e. in the framework of the corresponding S-model; so (2) the accepted statement in the given scientific theory treated in itself does not give any real information about the corresponding fragment of the real world which serves as the subject of the given theory. However, (3) if it is demonstrated that the S-model representation of the given scientific theory is correct in respect to its subject, i.e. that the m-relation of the given object-system to the given modelling system representing the given scientific theory really takes place, then the accepted statements in it, i.e. the theoretically (in a rational way) obtained statements in the given S-model obtain the value of expressing objective truth; in other words, they obtain the function to give information about the reality. So, the cognitive function of accepted theoretical statements in a given scientific theory, in particular of the theoretical laws of nature formulated 
in it, can be realised only through correspondence of the theory treated as an S-model of a fragment of reality as a whole to its subject, respectively to the object-system. In other words, the accepted theoretical statements of a given scientific theory, in particular the theoretical laws of nature formulated in it, correlate with the reality through the correlation of the corresponding theoretical S-model to the reality. Besides, (4) in the S-models and at the same time in human knowledge, in particular in scientific knowledge (treated as a part and type of human knowledge), objective value can possess the structure and at the same time the relations forming the given structure, but not the nature of initial elements taking part in construction of the given S-model; hence the knowledge of the nature of substratum of the real things and phenomena, in principle, can be obtained to some degree only by means of transition to S-modelling on the lower level of its object-system.

Note to Th. 7: Tr in S-Mdl. One may say that if the system $\mathbf{Y}$ contains the S-model of system $\mathbf{X}$, or in the particular case that human knowledge contains knowledge of a given fragment of the reality then something is transferred from the system-object $\mathbf{X}$ into the system-model $\mathbf{Y}$, respectively from the given fragment of the reality into human knowledge. This "something" is not the initial entities or processes which are the deepest bearers (respectively, a substratum) of the relations and structures of the system-object $\mathbf{X}$, however it is the structure or abstract relations in the system-object $\mathbf{X}$. So, our knowledge of the entities and processes can be obtained by means of transfer only of abstract relations from the reality to human knowledge.

From this epistemological statement follows a very important methodological conclusion: indeed, it is good for our knowledge to construct and to have such an S-model of the studied fragment of reality in which its image would harmonize with our visual perception of the same fragment of reality. However, it is much better for scientific knowledge to construct and to have such an S-model of the studied fragment of reality which well reproduces fundamental abstract relations and structures of the system-object, nevertheless the image of the latter in the S-model may have a discrepancy with our visual perception of the system-object. 


\subsection{System of Successive Spaces (SSS): a unified means of modelling of mathematical systems}

1) The System of Successive Spaces in its general form is introduced here in search of (1) a universal means for construction of S-models of a variety of mathematical systems on a general unified basis, (2) which reproduces their fundamental abstract relations and structures in an appropriately convenient form for scientific research (as it is required in the note to Th. 7 above) (3) with an intention for their application of various systems as well as processes studied in various natural sciences to S-modelling.

2) The term 'System of Successive Spaces' will be often abbreviated as 'SSS'. The same abbreviation in bold font will be used also in formal expressions.

3) The general intuitive idea of the concept of SSS may be expressed on an intuitive level in the following way:

3.1) Let us suppose that the space $\Omega$ is a linearly ordered set of separate subspaces $\Omega^{\mathbf{p}}, \mathbf{p} \in \mathbf{N}_{1}$, where $\mathbf{N}_{1}$ means the sequence of natural numbers beginning with 1 and directed to greater numbers (so, for $\mathbf{m}, \mathbf{n} \in \mathbf{N}_{1}, \mathbf{n}$ follows $\mathbf{m}$ if and only if $\mathbf{m}<\mathbf{n}$ ). These separate subspaces will be called partial spaces.

The set of all elements $\mathbf{a}^{\mathrm{p}}$ of all partial spaces $\Omega^{\mathrm{p}}, \mathbf{p} \in \mathbf{N}_{1}, \mathbf{i} \in \mathbf{N}_{1}{ }^{(\mathbf{p})}\left(\mathbf{N}_{1}\right.$ is the set of indexes of the partial spaces $\Omega^{\mathrm{p}}$ and $\mathbf{N}_{1}{ }^{(\mathrm{p})}$ is the set of indexes of the elements in a given partial space $\mathbf{p}$; in both cases $\mathbf{N}_{1}$ denotes the natural order of integers beginning with $1: 1,2,3, \ldots$ ), will be called 'universal space' and denoted by ' $\Omega$ ':

$\Omega=\mathrm{U} \Omega^{\mathrm{p}}$, besides $(\mathrm{q} \neq \mathrm{s}) \rightarrow\left(\Omega^{\mathrm{q}} \cap \Omega^{\mathrm{s}}=\varnothing\right), \mathrm{q}, \mathrm{s} \in \mathrm{N}_{1}$,

So, in such a way we have introduced a countable ordered set of successive (partial) spaces $\left\langle\Omega^{\mathrm{p}}>, \mathbf{p} \in \mathbf{N}_{1}\right.$, which will be abbreviated as 'SS-set'. In order to stress the fact that the SS-set $\Omega$ is an ordered set of separate partial spaces (cf. $\{8\}$ ) we shall denote it by $\Omega<$. Using the generally accepted rules of denotation in the set theory and in the classical propositional logic we may define an SS-set $\Omega<$ in symbolic way as follows:

$$
\Omega<\leftrightarrow_{\mathrm{Df}}\left(\left(\Omega=\mathrm{U} \Omega^{\mathrm{p}}\right) \&\left((\mathrm{q} \neq \mathrm{r}) \rightarrow\left(\Omega^{\mathrm{q}} \cap \Omega^{\mathrm{r}}=\varnothing\right)\right) \&\left(\left\{\Omega^{\mathrm{s}}\right\}=<\Omega^{\mathrm{s}}>\right)\right), \mathrm{p}, \mathrm{q}, \mathrm{r}, \mathrm{s} \in \mathrm{N}_{1},
$$

i.e. the set of successive spaces $\Omega<$ is a set of partial spaces which is ordered and countable. 
The pair of universal space and the ordered set of all partial spaces of the elements of which it is constituted may be treated as an elementary system with the simplest structure. So, we have:

$\Omega<=\left\{\Omega,<\Omega^{p}>\right\}$,

where the universal space $\Omega$ is the set-basis of the given SS-set $\Omega$, and the countable ordered set of all partial spaces $<\Omega^{\mathrm{p}}>$ is its structure.

Obviously, the following conditions for the set of successive spaces, formulated below as postulates P1-P3, are satisfied:

P $[$ SS-set $]$ 1. $\Omega=\mathbf{U} \Omega^{\mathrm{p}}$, where $\mathbf{p} \in \mathbf{N}_{1}$,

P[SS-set $] 2 .(\mathbf{p} \neq \mathbf{q}) \rightarrow\left(\Omega^{\mathrm{p}} \cap \Omega^{\mathrm{q}}=\varnothing\right), \mathbf{p}, \mathbf{q} \in \mathbf{N}_{1}$,

$\mathbf{P}\left[\right.$ SS-set]3. $(\mathbf{p}<\mathbf{q}) \rightarrow\left(\Omega^{\mathrm{p}} \Rightarrow \Omega^{\mathrm{q}}\right)$, where $\mathbf{i}, \mathbf{j} \in \mathbf{N} \mathbf{1}$, "a $\Rightarrow \mathbf{b}$ " means "b follows a".

3.2) A set of successive spaces in itself may hardly be of pragmatic interest; however, we consider it to be significant from a logical point of view as a basis for construction of various more complicated systems of successive spaces possessing considerable theoretical and pragmatic significance and so this circumstance, in particular, gives the study of SS-sets indirect theoretical as well as pragmatic significance. The transition from sets of successive spaces (SS-sets) to various kinds of more complicated systems of successive spaces (SSS) may be realised by means of supplying of the ordered set of different partial spaces $\left\langle\Omega^{\mathrm{p}}\right\rangle$, with some kind of connections between elements or sets of different partial spaces called 'succession(s)' and specified in an axiomatic way. The successions are constructible from the most elementary successions called ribs-connection between two points $\mathbf{a}_{\mathbf{i}}^{\mathbf{p}}$ and $\mathbf{b}^{\mathbf{p}+1}{ }_{\mathbf{i}}$ in two different adjacent partial spaces $\Omega^{\mathrm{p}}$ and $\Omega^{\mathrm{p}+1}$, respectively $\mathbf{a}^{\mathrm{p}}{ }_{\mathrm{i}} \in \Omega^{\mathrm{p}}, \mathbf{b}^{\mathrm{p}+1}{ }_{\mathrm{j}} \in \Omega^{\mathrm{p}+1}$. The systems obtained in such a way are graphs of a giving type specified as 'K-graphs' and the basic qualitative types of formal relations expressed by them will be called 'K-relations'. A more precise term for this type of systems constructed on the SS-sets $\Omega<$ is 'System of Successive Spaces of the first order', abbreviated as 'SSS1', and denoted in formal expressions as 'SSS1'.

The SSSS1 may be presented and at the same time defined as a triple of the following way: 
SSS1 $=\left\{\Omega,\left\langle\Omega^{\mathrm{p}}>,\left\{\mathbf{u}_{\mathrm{s}}\right\}\right.\right.$, where $\mathbf{p}, \mathbf{s} \in \mathbf{N}_{\mathbf{1}}, \mathbf{u}_{\mathbf{s}}$ denotes $\mathbf{s}$-succession on $<\Omega^{\mathrm{p}}>$,

provided $\Omega \neq \varnothing,\left\{\mathbf{u}_{\mathrm{s}}\right\} \neq \varnothing, \mathbf{p}>1$, where the universal space $\Omega$ is the set-basis of the given SSS, and the ordered pair $\left\langle\left\langle\Omega^{i}\right\rangle,\left\{\mathbf{u}_{j}\right\}\right\rangle$ is its structure.

The K-relations in SSS1 mentioned above can be applied as S-models of various types of relations of general kind, as for example:

3.2.1) Relations of correspondence from which are obtainable relations of quantitative comparisons like more, less, equal as well as various forms of $S$-modelling relations;

3.2.2) Relations between (1) substantives, i.e. bearers of properties and relations, and (2) properties;

3.2.3) Relations between (1) previous and (2) next moment of time in a discrete process;

3.2.4) Relations between (1) the factor and (2) the result in a relation of determination in a very general sense embracing logical relations between premises and consequence as well as physical relation between cause and effect.

3.2.3*) If the ordered set of partial spaces $<\Omega^{\mathrm{p}}>$ is continuous, then $\mathrm{K}$-relations in SSS1 can be applied as S-models of relations between (1) previous and (2) next moment of time in a continuous (in distinction of the case mentioned above under point 3.2.3) process.

Obviously, the SS-set can be treated as a system of successive spaces for which $\left\{\mathbf{u}_{\mathrm{s}}\right\}=\varnothing$, correspondingly, as a System of Successive Spaces of the zero degree and denoted as 'SSSO'.

3.3) If a definite metrics is given on the various partial spaces we can treat them as coordinates and thus we obtain a system of parallel coordinates. The system of this kind will be called the 'System of Successive Spaces of the second order', abbreviated as 'SSS2', and denoted in formal expressions as 'SSS2'.

The SSS2 may be defined as a quadruplet in the following way:

SSS2 $=\left\{\Omega,<\Omega^{\mathrm{p}}>, \mu\left(\Omega^{\mathrm{p}}\right),\left\{\mathbf{u}_{\mathrm{s}}\right\}\right\}$,

where $\mathbf{p}, \boldsymbol{s} \in \mathbf{N}_{1}, \mu\left(\Omega^{p}\right)$ denotes metrics on the partial space (respectively, system of coordinates) $\Omega^{\mathrm{p}}$, $\mathbf{u}_{\mathbf{s}}$ denotes $\boldsymbol{s}$-succession on $\left\langle\Omega^{\mathrm{p}}>\right.$. In $\{15\}$ 
the universal space $\Omega$ is the set-basis of the given SSS2, and the ordered triplet $\left\langle\left\langle\Omega^{\mathrm{P}}>, \mu\left(\Omega^{\mathrm{P}}\right),\left\{\mathbf{u}_{s}\right\}>\right.\right.$ is its structure.

The coordinate system, initially used by Hipparchus in the $2^{\text {nd }}$ century B.C. in a concrete geographical form as a way to map places on earth using latitudes and longitudes (cf. Rouse Ball, 1908; 1960, p. 88), was factually introduced in geometry by P. Fermat (1636) and especially by R. Descartes (1637), in essence as a unified means of algebraic modelling of various kinds of lines. The very beginning of the first book of the Descartes's monograph reads:

Any problem in geometry can easily be reduced to such terms that a knowledge of the lengths of certain straight lines is sufficient for its construction. Just as arithmetic consists of only four or five operations, namely, addition, subtraction, multiplication, division and the extraction of roots, which may be considered a kind of division, so in geometry, to find required lines it is merely necessary to add or subtract other lines... I shall not hesitate to introduce these arithmetical terms into geometry, for the sake of greater clearness.

Often it is not necessary thus to draw the lines on paper, but it is sufficient to designate each by a single letter... Here it must be observed that by $\boldsymbol{a}, \boldsymbol{b}$, and similar expressions, I ordinarily mean only simple lines, which, however, I name squares, cubes, etc., so that I may make use of the terms employed in algebra. (Descartes, 1925, pp. 2-5; pp. 297-299 in the original French text)

As a result of applying such an approach to establishing a connection between geometry and algebra factually based on the use of the coordinate system, the primary elements of analytical geometry were laid down in the two books mentioned above. However, already in the third, and final, book of the same monograph, dedicated to the solution of some algebraic problems, the same connection is used in a rather opposite direction—for geometrical modelling of algebraic equations.

So, the coordinate system, applied in Descartes's La Geometrie in a really implicit, but very effective form, factually functioned as a unified means of modelling of mathematical systems for both main parts of mathematics of that time- the algebra and the geometry. In the course of development of mathematics after Descartes, the coordinate system and the way it was applied were considerably 
improved, obtaining various new forms in accordance with the appearance of various new kinds of mathematical systems and new research problems. As a result, the forms, domain and the role of the coordinate systems in mathematics have increased. One can treat the present proposal of SSS2 as a parallel coordinate system to be merely an element of the mentioned process of increase of the forms of coordinate systems. Besides, there are some advantages of the parallel coordinate system over the Cartesian one in which the intersection of all coordinate lines in one and the same point, called the origin, takes place. A point in n-dimensional space for $\mathbf{n} \in \mathbf{N}_{\mathbf{1}}, \mathbf{n}>\mathbf{3}$, as well as the corresponding vector of $\mathbf{n}$ components, can be represented effectively as a (usually broken) line connecting one by one point for each partial space $\Omega^{\mathbf{p}}, 1 \leq \mathbf{p} \geq \mathbf{n}$ (see Figs. 1-10 in Chendov, 1998, pp. 25-27). Moreover, in distinction from the Cartesian coordinate system in the parallel one it is possible to speak about continuousdimensional space-the ordered set $\left\langle\Omega^{\mathrm{p}}\right\rangle$ is continuous and the point can be represented in some cases by means of a continuous line in $\left\langle\Omega^{\mathrm{p}}\right\rangle$, respectively by means of a corresponding continuous function.

\section{Abstract structures of the Logic of Science: initial steps of their elaboration}

\section{Preliminary notes}

The first step towards elaboration of the abstract structures of logic of science consists in realisation of the first step in the process of elucidating the content of the basic concept (respectively, of the sense of basic term) in the formulation of the present topic of research: the 'logic of science', introduced in Thesis 4, point 2 as a foundational complex science of the first order (in the sense formulated under point 2.2.1), which contains an essential part of the most important fundamental theoretical productive factors for elaboration of the multiformity of interdisciplinary sciences and theories. Besides, one has to take into account the historic-empirical fact that the logic of science as a complex theory appears as a result of the historical development of logic as a canonical theory. Thus, first of all, we have to raise the question about the content of concept (respectively, about the meaning of the term) 'logic' in a form appropriate for methodological research, similarly to the way in which Dedekind raised the question about the nature of numbers (see Dedekind, 1888) — What is logic and what should it be? 


\subsection{What is logic and what it should be?}

\subsubsection{What logic was and what it is?}

Tendencies of the historical development of logic

1) Logic originates in ancient Greece during the $4^{\text {th }}$ century B.C. in the Megarian school of philosophy and especially in the works of Aristotle in Athens, chiefly in Book I of Prior Analytics, generally speaking as a theory of logical inference. Even though his works, which served as a point of departure for the historical development of the logic, are preserved, we cannot find in them a clear concept of logic which could serve as a point of departure for the historical substantiation of a contemporary concept of logic. Therefore, in view of such a purpose, it is important to determine namely which of his works served as a point of departure for the historical development of the logic.

The very beginning of Prior Analytics reads: "First we must state the subject of the inquiry and what it is about. The subject is demonstration, and it is about demonstrative understanding." (Aristotle, 1984, p. 39)

Later on Aristotle makes a very significant distinction between the concepts 'demonstration' and 'deduction' (respectively, 'syllogism') and expressly states the following relation between them: "Deduction should be discussed before demonstration, because deduction is more general: a demonstration is a sort of deduction, but not every deduction is a demonstration" (Aristotle, 1984, p. 41).

In correspondence with this statement in Book I of Prior Analytics, Aristotle devotes his attention to the elaboration of the theory of deduction, respectively of syllogism, and later on, in Book II of Prior Analytics he devotes his attention to the elaboration of the theory of demonstration on the basis of the already elaborated theory of deduction (syllogism). Using contemporary terminology, we can say that in Posterior Analytics, Aristotle devotes his attention to the elaboration of the methodology of science on the basis of his theory of demonstration and at the same time of his theory of deduction, respectively of syllogism. So, the following triplet of fundamental scientific theories takes place in Aristotle's Analytics:

$<$ Syllogistic, resp. - Theory of Demonstration - Methodology of Science> Theory of Deduction
Pr. An., Book I
Pr. An., Book II
Post. An. 
We find in Aristotle's works also some considerations about induction and its role in human knowledge.

In the historical process of following development of logic, in which it takes a more or less definite shape, namely the Aristotelian syllogistic, chiefly his assertoric syllogistic (as distinctive from the modal one), has played the main role. In this connection, we can add to the last one also the role of various his statements in his various works concerning propositions, which, using a modern terminology, can be treated in their totality as a fragment of the logic of propositions.

Actually, a well-elaborated logic of propositions with expressly formulated rules of inference was produced in the Megarian-stoic logical school during the $4^{\text {th }}$ and $3^{\text {rd }}$ centuries B.C.

2) Afterwards, logic was developed in various directions, and in parallel to this process, its concept has obtained various specifications. In addition, a deep, historically significant, new treatment of the logic was proposed by Leibniz in the second half of the $17^{\text {th }}$ century and the beginning of the $18^{\text {th }}$ century. Here we shall limit ourselves to mentioning his idea and programme of mathematisation of logic, i.e. to apply mathematical means for treatment of logical problems and accentuating at the same time the significance of logic for scientific knowledge (see Leibniz, 1960). This makes him the pioneer of mathematical logic. Following Leibniz's idea and the programme just mentioned, George Boole initiated the development of the algebra of logic as an initial variant of the classical propositional logic; later Gottlob Frege elaborated the formal axiomatic system of the classical propositional logic and on its basis constructed formalised logic of many-placed predicates as a considerable generalisation of Aristotle's syllogistic, by analogy with the mathematically well-elaborated concept of function of many arguments, and in a form which allowed to express the relations in the mathematical discourse. In such a way, Frege found a way to apply logic to mathematics, more precisely, to draw the arithmetic of natural numbers from the logic of predicates.

3) During the last three decades of the $19^{\text {th }}$ century and the first three decades of the $20^{\text {th }}$ century, the three basic schools in the foundations of mathematics and at the same time in the philosophy of mathematics—logicism (Frege, Russell, Whitehead), intuitionism (Brouwer, Weyl, Heyting), and formalism (Hilbert, Bernays)—proposed three different conceptions concerning the relations between logic and mathematics. Every one of these three schools has contributed the development of logic as well as considerably strengthened 
the connection between logic and mathematics. As a result of the latter, more and more scholars have accepted the so-called mathematical logic to be the only contemporary logic and, at the same time, the logic to be a part of mathematics.

4) After the publication of the fundamental three-volume work Principia Mathematica by Whitehead and Russell (1910-1913), a new stage of the history of logic began to change shape considerably: (1) various types of logical systems proposing the treatment of, correspondingly, various kinds of logical inference and obtaining the characteristic "non-classical" (in distinction from classical propositional and predicate logics) are coming into being; (2) in some of the non-classical systems of logic, the values 'true' and 'false' as well as their strengthening or weakening, like modal ones, are not applicable-for example, the logic of question, the logic of tasks, the logic of prescription (as a particular case, deontic logic), etc.; (3) the logic has obtained applications outside mathematics—in theoretical physics, biology, technology and so on; (4) the structure of a logical system has significantly changed by means of express distinction between (4.1) logical syntax, respectively formal logical system, logical calculation, (4.2) logical semantics, and (4.3) metalogical system; (5) more and more application of models in logical semantics took place, so that it seems that some logicians even associate logical semantics with logical modelling theory; etc.

\subsubsection{What logic should be?}

The basic elements of the General Theory of Logical Systems

In view of (1) the facts of the history of logic, outlining definite tendency of its development starting from the field of philosophy towards the field of mathematics, (2) fundamental change in the subject of mathematics in direction from a deductively substantiated science of numbers and figures, i.e. of quantitative and spatial relations, into deductively substantiated science of abstract relations, structures and systems, and (3) some considerations of general philosophicomethodological character in the spirit of interdisciplinary positivism manifested in Chendov, 2013 the following principal theses and definitions are accepted here, in their totality treated as a basis of the General Theory of Logical Systems:

1) Thesis 1. The logic (as a science) is an hierarchical system of atomic logical systems - a set (respectively, a combination) of two or more atomic logical systems, or a construction of atomic logical system on the basis of an atomic logical system produces a complicated logical system of the first degree; a 
set (respectively, a combination) of a given complicated logical system of the first degree together with other logical system(s) of the first degree or atomic ones as well as a construction of atomic logical system on the basis of a logical system of the first degree produces a complicated logical system of second degree; and so on.

Note to Thesis 1. It follows immediately from Thesis 1 that, generally speaking, logic is a set of various logical systems, besides logic in a well systematised form is a system of logical systems.

2) Definition 1 (preliminary definition of the concept 'logical system'). A system is called logical if and only if it reveals properties of a given type of the relation of logical inference, treated on syntactical, semantic and metalogical (metamathematical) levels.

Note 1 to Definition 1. The content of Definition 1 can be expressed otherwise in the following way: a logical system of a given type is a theoretical system of logical inference of the correspondingly given type.

Note 2 to Definition 1. The content of Section 2.1.1 above gives a historico-empirical substantiation of this definition: indeed, namely the treatment of a type of logical inference as syllogisms by Aristotle and the rules of inference for propositions in the Megarian-stoic logical school played the main role as a historical source for origination and further development of logic, see Section 2.1.1, point 1), as well as the various contemporary logical systems are specified as theoretical systems of treatment of correspondingly different types of logical inference, see Section 2.1.1, point 4) and (1).

Note 3 to Definition 1. Definition 1 presupposes the following statement formulated below as a thesis 2:

3) Thesis 2 . There is a variety of types of logical inference, and, in addition, one and the same type of logical inference may belong to several formal logical systems (1) distinguishable by means of the corresponding sets of derived formulas, (2) as its various explications.

Example to Thesis 2. C. I. Lewis's monadic modal systems S1-S5 constructed in Lewis and Langford, 1959, are logical systems of one and the same type; the systems S1-S5 propose five different formal explications of the concept 'modal logical inference of Lewis's type'.

4) Thesis 3. To every type of logical inference corresponds a type of logical system and vice versa. 
Argumentation. Thesis 3 follows immediately from Definition 1: "and vice versa" in the formulation of Thesis 3 results from "if and only if" in Definition 1.

Note to Thesis 3. In view of the correspondence between the type of logical inference and the type of logical system it is natural both to be named by means of using one and the same specifying term. For example, from the specifying term 'dyadic modal' the terms 'dyadic modal inference' and 'dyadic modal system' can be derived.

Note to Definition 1 and Theses 1-3. In view of Definition 1 and Theses 1-3 we have to reduce the task to state a general concept of logic (as a science), revealing its subject of study, to the task to state a general concept of logical inference, i.e. to state a concept of the relation by which from a given presupposition(s) one can derive a given conclusion in the most general form. The last task is realised next, in Thesis 4 .

5) Thesis 4 . The concept of logical inference as well as the corresponding concept of logical system in the most general sense concern logical acceptance and logical non-acceptance.

Language convention to Thesis 4 . The terms 'acceptance' and 'nonacceptance' are used here in the following two basic, more concrete forms:

as values: ' $\rightarrow \rightarrow_{i} \mathrm{~A}$ ' denotes ' $\mathbf{A}$ is $\mathbf{i}$-accepted', respectively ' $\mathbf{A}$ is accepted in $\mathbf{i}$-form', or ' $\mathbf{A}$ is accepted in $\mathbf{i}$-way';

as relations (in the simplest form): ' $\left(\rightarrow_{i} A\right) \rightarrow_{j} \mathbf{B}$ ' denotes 'i-acceptance of $\mathbf{A}$ entails $\mathbf{j}$-acceptance of $\mathbf{B}$ ', otherwise speaking 'if $\mathbf{A}$ is $\mathbf{i}$-accepted, then $\mathbf{B}$ is $\mathbf{j}$-accepted'.

If the terms 'acceptance' and 'non-acceptance' are used as values or relations in the sense of the above points 1) and 2), besides $\mathbf{A}, \mathbf{B}$, etc. can be various elements of thought as well as of the objective reality outside the thought, then in order to specify such a sense of them I shall use the terms 'logical acceptance' and 'logical non-acceptance', respectively.

Note to the language convention to Thesis 4. From the assertion "A, B, etc. can be various elements of thought as well as of the objective reality outside the thought" in the basic text of the language convention to Thesis 4 it follows that the terms 'acceptance' and 'non-acceptance' in both basic, more concrete forms - as values and as relations, are used here in a very general sense which as with the case of the concept 
'information' in contemporary mathematics is including both kinds of aspects—epistemic and ontological—i.e. we can speak that something is accepted or not accepted by human mind but also by a natural agent.

6) Definition 2 (the final definition of the concept 'logical system'). Logical system is (1) a system (2) of theoretical character (3) whose subject-matter of study is the logical acceptance of a definite type determining its specification, (4) treated (4.1) on a proper level in (4.1.1) syntactical, (4.1.2) abstract semantic, and (4.1.3) specific logico-semantic ways, as well as (4.2) on the metalogical level.

Taking into account the note to Thesis 1 and Definitions 1 and 2 we can accept the following Definition 3-the final definition of the concept 'logic' (as a science) in two forms: abbreviated and enlarged:

Definition 3.1 (the final definition of the concept 'logic' in abbreviated form). Logic is a mathematical science of logical acceptance (in its pure form).

Definition 3.2 (the final definition of the concept 'logic' in enlarged form). Logic is (1) a science of (2) abstract (mathematical) structure of logical acceptance (3) treated (3.1) on a proper level in (3.1.1) syntactical, (3.1.2) abstract semantic and (3.1.3) specific logico-semantic ways, as well as (3.2) on metalogical level.

7) Thesis 5. (1) The concept of logical inference as well as (2) the corresponding concept of logical system in the most general sense as concerning logical acceptance and logical non-acceptance (in accordance with the above Thesis 4) and (3) the concept of logic as a mathematical science of logical acceptance (in accordance with Definition 3) are more general than the concepts of $\left(1^{*}\right)$ logical inference, $\left(2^{*}\right)$ logical system and $\left(3^{*}\right)$ logic as a science, usually accepted by contemporary logicians.

In other words, all cases of accepted treatment in the contemporary logic of the concepts 'logical inference', 'logical system' and 'logic' (as a science) allow the corresponding treatment by means of the concepts 'logical inference', 'logical system' and 'logic' treated as concerning logical acceptance and logical non-acceptance; however, the concepts 'logical inference', 'logical system' and 'logic' treated as concerning logical acceptance and logical nonacceptance allow such cases which are not acceptable from the usual point of view in contemporary logic.

Examples to Thesis 5. The values 'true' and 'false' in classical propositional logic and in classical predicate logic can be treated as 
a particular case of 'acceptance' and 'non-acceptance' as values: a given proposition to be accepted or non-accepted as true; the material implication ' $\mathbf{p} \rightarrow \mathbf{q}$ ' can be treated as a particular case of relation acceptance in the following form: "if $\mathbf{p}$ is accepted to be true, then $\mathbf{q}$ is accepted to be true", or "the acceptance of $\mathbf{p}$ as true entails acceptance of $\mathbf{q}$ as true", naturally in accordance with the table of true-values for material implication.

The scheme of modus ponens can be generalized for logical acceptance in the following way:

$$
\frac{\left\{\rightarrow_{i} \mathrm{~A}_{u}\right\},\left\{\rightarrow_{i} \mathrm{~A}_{u}\right\} \rightarrow_{j} \mathbf{B}}{\rightarrow_{j} \mathbf{B}}
$$

The classical propositional calculus as constructed in Hilbert and Ackermann (1928) can be treated as a complicated logical system of the first degree in the sense of Thesis 1: (1) the definition of the concept formula can be treated as a construction of initial atomic logical system concerning acceptance of something as a formula and (2) the construction of the proper system of propositional calculus by means of four axioms and two rules of inferenceas an atomic logical system concerning acceptance of something as a derived formula which is a superstructure over the first logical system mentioned above (see point 1). Further, the classical predicate calculus can be thus treated as a complicated logical system of third degree.

8) Thesis 6. (1) The wide-spread statement that the laws and forms of correct thought are the subject-matter of logic, i.e. that logic is a science concerning correct thought, is not correct. In accordance with the definition formulated above, the subject-matter of logic is not thought at all, respectively this or some other aspect of it, but an abstract mathematical object like sets, graphs, functions, numbers, vectors, tensors, and so on-namely, the relation of logical inference concerning logical acceptance. Hence, logic, taking shape since the $17^{\text {th }}$ century chiefly in the works of Leibnitz, Bool, Frege, Russell and Hilbert, has become a mathematical discipline, i.e. a part of mathematics, like and together with algebra, topology, mathematical analysis, probability theory, etc. (2) At the same time we have to take into consideration the historic-empirical fact that correct thought is essentially involved in the scope of applications of logic, moreover, namely it has and still continues to play a significant heuristic role for the formation of the initial ideas obtaining later theoretical elaboration in logic. 
Note to Section 2.1.2. The treatment of the subject of logic in some way by means of the concept 'acceptance' can be found also in publications of some other authors (cf. Aho, 1994). The conception of logic formulated above in the proper text of section 2.1.2 is the result of a long process of deliberation by the author of present paper on the question "What is logic?" in the course of the treatment of various logical problems in close relation with the application of logic to various branches of science, besides various elements of this conception were already published.

\subsection{On explication of the concept 'logic of science'}

2.2.1 On the basis of the methodological analysis of the concept 'logical system' and taking into account empirical facts related to the history and present state of applications of logic in various branches of science I accept the following definition of logic of science:

Definition 5.1 (abbreviated form). Logic of science is a complex science of acceptance-relation (i.e. of logical inference in the most general sense), including philosophico-methodological, proper-logical, historical, and (emphatic) scientific-applied aspects in their mutual connection.

Definition 5.2 (enlarged form). Logic of science is a complex science of logical inference, integrating in their mutual relationship the following three types (resp. classes) of problems:

1) proper problems of logical systems in a volume and form, relevant to their applicability;

2) problems of applicability as well as of application of the logical systems to various branches of the science as a tool for (1) research of separate scientific problems, (2) semantic elucidations of abstract theoretical concepts and statements, (3) substantiation of scientific theories and (4) S-modelling in scientific theories on both levels-(4.1) epistemic (i.e. full or partial S-modelling of the given theoretical systems) and (4.2) ontological (i.e. full or partial S-modelling of real systems and processes which are subject of study of the given theoretical system);

3) philosophico-methodological problems about the (3.1) foundations, (3.2) elaboration and (3.3) applications of the logical systems in their mutual connections. 
Note 1 to Definition 5.2. Namely the significance of the application of logic to logical substantiation of the set theory and of S-modelling methodology (as a particular case of realisation of its function mentioned in Definition 5.2, point 2), (3)) which in their naïve (non-logical) form are presupposed from its elaboration, is the reason for unifying both complex theories of the first degree S-modelling methodology and logic of science in a complex theory of the second degree called 'logistics'.

Note 2 to Definition 5.2. The statement in Definition 5.2, point 2), (4.2) is essentially based on the treatment of logic as a science of logical acceptance allowing ontological interpretations.

2.2.2 It is especially worth stressing that the relation between the three types of problems mentioned in Definition 5.2 is not merely an enrichment of the logic as a canonical science by means of addition with problems about its applications and with ones of philosophico-methodological character, but it is manifestation of the interdisciplinary approach to the investigation of scientific problems, so, it involves also a retrospective influence of the latter upon the treatment of the logical problems.

\section{Closing words}

The initial (methodological) statements concerning the abstract structures of S-modelling theory and logic of science in Sections 1 and 2 of the present paper are necessarily formulated in a very general form. In order to make them perfectly clear it is necessary to realise them on a more concrete level by means of elaboration of the technical aspect of their abstract structures and further by means of applications of the latter to mathematics and natural sciences. However, this task which naturally arises from the considerations in Sections 1 and 2, goes out of the task formulated at the very outset of the present paper; consequently its development has reached its logical end. 
An Approach to Abstract Structures of Logistics as a Complex Theory Unifying the Methodology of S-Modelling and the Logic of Science: Initial Steps

\section{References}

Aho, T. (1994), On the Philosophy of Attitude Logic, Acta Philosophica Fennica, vol. 57, Helsinki: Societas Philosophica Fennica.

Aristotle (1984), Prior Analytics in J. Barnes (ed.) The Complete Works of Aristotle, vol. I, Princeton, NJ: Princeton University Press.

Beth, E. (1968), The Foundations of Mathematics: A Study in the Philosophy of Science, New York: Harper \& Row.

Chendov, B. (1998), 'System of successive spaces (SSS) - a unified means of modelling of mathematical systems,' in B. Chendov \& T. Mikhajlova (eds.) Methodology of Mathematical Modelling, vol. VI, Sofia.

- (2013), 'Interdisciplinary Positivism - Third Stage of Development of Positivistic Philosophy,' in XXIII World Congress of Philosophy, Athens 04-10 August, 2013: Abstracts, p. 114.

Dedekind, R. (1888), Was sind und was sollen die Zahlen? Braunschweig: F. Vieweg und Sohn.

Descartes, R. (1925[1637]), The Geometry (with a facsimile of the first edition), New York: Dover Publications, Inc. [Original edition (1637), La Geometrie, Leyde: Jan Maire]

Hilbert, D. (1899), Grundlagen der Geometrie, Leipzig: B. G. Teubner.

_ (1905), 'Über die Grundlagen der Logik und der Arithmetik,' in Verhandlungen des Dritten Internationalen Mathematiker-Kongresses in Heidelberg, vom 8. bis 13. August 1904, Leipzig: B. G. Teubner.

(1922), 'Neu Begründung der Mathematik,' in Abhandlungen aus dem mathematischen Seminar der Hamburgschen Universität, vol. 1.

(1923), 'Die logischen Grundlagen der Mathematik,' in Mathematische Annalen, vol. 88 .

(1928), 'Die Grundlagen der Mathematik,' in Abhandlungen aus dem mathematischen Seminar der Hamburgschen Universität, vol. 6; in Hamburger Mathematische Einzelschriften, vol. 5, Leipzig: B. G. Teubner.

- (1929), 'Probleme der Grundlegung der Mathematik', in Atti del Congresso internazionale dei matematici, Bologna, 3-10 settembre 1928, vol. 1, Bologna.

Hilbert, D. \& Ackermann, W. (1928), Grundzüge der theoretischen Logik, Berlin: Springer.

Hilbert, D. \& Bernays, P. (1934, 1939), Grundlagen der Mathematik, B. I \& II, Berlin: Springer-Verlag.

Leibniz, G. (1960), Fragmente zur Logik, Berlin: Akademie-Verlag.

Lewis, C. I. \& Langford, C. H. (1959[1932]), Symbolic Logic, $2^{\text {nd }}$ edition, New York: Dover Publications, Inc. 
Neurath, O.; Carnap, R. \& Morris, Ch., eds. (1939; 1969), Foundations of the Unity of Science: Towards an International Encyclopedia of Unified Science, vol. I, Chicago \& London: The University of Chicago Press.

Boris Chendov earned the Doctor of Philosophical Sciences (Dr. phil. sci.) degree for his thesis 'Logical Systems and Modelling of Indefiniteness', at the Moscow State University in 1994. Right before that, he was research professor in Brussels at the centre chaired by Ilya Prigogine. Boris Chendov has done research on philosophy of science, in particilar on philosophy of mathematics and physicas, as well as in field of logic and history of foundations of science. Professor Chendov is the initiator and organiser of interdisciplinary research in Bulgaria. He has acted as chair of various research groups and projects mostly at the Institute of Philosophy of the Bulgarian Academy of Sciences. He has contributed to numerous international conferences as a speaker and symposia organiser. Boris Chendov is a member of several academies and scientific societies in Italy, Russia and Ukraine. Currently, he is organising an International Institute of Foundational, Historical and Interdisciplinary Problems of Science. In addition to his home country Bulgaria, Professor Chendov has given lectures at outstanding universities in the U.S.A., Germany, Russia and China. He has been involved in publishing as an author, editor, editorial board member and reviewer in numerous cases. 